\title{
On the papers of Ramachandra and Kátai
}

\author{
by
}

\section{A. Sankaranarayanan and K. Srinivas (Bombay)}

1. Introduction. In [2] K. Ramachandra proved the following general result. Let $S_{1}$ denote the set of all $L$-series. We can define $\log L(s, \chi)$ for $\operatorname{Re} s>1$ by the series

$$
\sum_{m} \sum_{p} \frac{\chi\left(p^{m}\right)}{m p^{m s}}
$$

where the sum over $m$ is over all positive integers and $p$ runs over all primes. For any complex constant $z$, we can define $(L(s, \chi))^{z}$ as $\exp (z \log L(s, \chi))$. Let $S_{2}$ consist of the set of all derivatives of $L(s, \chi)$ for all $L$-series and let $S_{3}$ denote the set of logarithms as defined above for all $L$-series.

Let $P_{1}(s)$ be any finite power product (with complex exponents) of functions of $S_{1}$. Let $P_{2}(s)$ be any finite power product (with non-negative integral exponents) of functions of $S_{2}$. Also let $P_{3}(s)$ denote any finite power product (with non-negative integral exponents) of functions of $S_{3}$. Let $c_{n}$ $(n=1,2,3, \ldots)$ be complex numbers which are $O_{\varepsilon}\left(n^{\varepsilon}\right)$ for every positive constant $\varepsilon$ and suppose that $F_{0}(s)=\sum_{n=1}^{\infty} c_{n} n^{-s}$ is absolutely convergent in $\operatorname{Re} s>1 / 2$. Finally, put

$$
F_{1}(s)=P_{1}(s) P_{2}(s) P_{3}(s) F_{0}(s)=\sum_{n=1}^{\infty} g_{n} n^{-s}
$$

and

$$
N_{1}(x)=\sum_{n \leq x} g_{n} \quad \text { for } x \geq 0 .
$$

Then we have

THEOREM 1. Let $x$ and $X$ be sufficiently large and $1 \leq h \leq x$. Consider a circle of positive radius ( $a$ constant depending only on $F_{1}(s)$ ) with 1 as centre which has no singularities of $F_{1}(s)$ (except possibly $s=1$ ) in its interior and on the boundary. From this circle remove the point of intersection with the real axis which lies to the left of 1 . Let $C_{0}$ denote the contour got by traversing the remaining portion of the circle in the anti-clockwise direction. 
Let

$$
I_{1}(x, h)=\frac{1}{2 \pi i} \int_{0}^{h}\left(\int_{C_{0}} F_{1}(s)(v+x)^{s-1} d s\right) d v .
$$

Then we have with $\varphi=1-1 / B+\varepsilon$ and $\varphi^{\prime}=1-2 / B+\varepsilon$

$$
N_{1}(x+h)-N_{1}(x)=I_{1}(x, h)+O_{\varepsilon}\left(h \exp \left(-(\log x)^{1 / 6}\right)+x^{\varphi}\right)
$$

and

$\frac{1}{X} \int_{X}^{2 X}\left|N_{1}(x+h)-N_{1}(x)-I_{1}(x, h)\right|^{2} d x=O_{\varepsilon}\left(h^{2} \exp \left(-(\log X)^{1 / 6}\right)+X^{2 \varphi^{\prime}}\right)$.

Here $B$ is the constant occurring in the density result

$$
N_{\chi}(\alpha, T)=O\left(T^{B(1-\alpha)}(\log T)^{200}\right)
$$

and $\varepsilon$ is an arbitrary small positive constant such that $\varphi$ and $\varphi^{\prime}$ are less than 1 .

This Theorem 1 includes as special cases the following two theorems due to A. E. Ingham and A. Selberg.

THEOREM 2. We have

$$
\pi(x+h)-\pi(x) \sim h(\log x)^{-1}
$$

where $h=x^{\varphi}$ with any constant $\varphi(5 / 8<\varphi<1)$.

Remark. Due to the "density results" of later writers, notably G. Halász, P. Turán, H. L. Montgomery and M. N. Huxley the constant $5 / 8$ has been sharpened and the latest $7 / 12$ is due to M. N. Huxley.

Theorem 3. We have, for $1 \leq h=h(X) \leq X(\log X)^{-A}$ (where $A$ is any constant $>10)$, and $\varphi^{\prime}>1 / 4$

$$
\frac{1}{X} \int_{X}^{2 X}\left(\pi(x+h)-\pi(x)-\frac{h}{\log X}\right)^{2} d x=O_{A}\left(h^{2}(\log X)^{-A}+X^{2 \varphi^{\prime}}\right) .
$$

Rem ark. Due to the "density results" of the authors mentioned above, the constant $1 / 4$ has been sharpened and the latest $1 / 6$ follows from Selberg's method on using Huxley's result. Ramachandra's result included the proper analogues for $\sum_{n \leq x} \mu(n)$ (in place of $\pi(x)$ ) which was new. Next in [1], I. Kátai used Ramachandra's result to obtain the uniform result

$$
\sum_{\omega(n)=k, x \leq n \leq x+h} 1=(1+o(1)) \frac{h(\log \log x)^{k-1}}{(\log x)(k-1) !}
$$

where $k$ is any positive integer (uniformly in $1 \leq k \leq \log \log x+$ $c_{x}(\log \log x)^{1 / 2}$ provided $c_{x} \rightarrow \infty$ sufficiently slowly $)$. 
In the present note, we sketch a uniform version of the general result of Ramachandra. The statement of the uniform result needs some preparations which we will take up in the next section. We state certain details in some generality in order that it may be useful to later workers.

2. Some preparations and the statement of the main theorem. We adopt the notation of [2] (as in [2] an important result used will be the zero-free region due to I. M. Vinogradov). We note that the results of [2] depend on the properties of $\zeta(s)$ and $L(s, \chi)$ only in $\sigma \geq 1 / 2$ and in particular do not depend on their functional equation. (On page 321 of [2] there is a reference to Ramachandra's paper on Titchmarsh's phenomenon and the functional equation. But we remark that in constructing the Hooley-Huxley contour, we may give to the points $\frac{1}{2} \pm i m(m=0,1,2, \ldots)$ the same treatment as was given to the zeros and this dispenses with the results referred to.)

Let $a_{n}=a_{n}(x)(n=1,2,3, \ldots)$ be a sequence of complex numbers depending on a real parameter $x \geq 10$. We assume that $a_{n}=O_{\eta}\left((n x)^{\eta}\right)$ for fixed $\eta>0$. The modified Hooley-Huxley contour is formed with respect to a non-empty finite set of $L$-functions of bounded moduli (we can to some extent relax this condition; but we do not consider such questions here) and with respect to parameters $a$ (a small constant depending on $\varepsilon$ ), $b=$ $1-(\log \log T)^{-1}$ and $\theta=1-(\log \log T)^{-3}$. Note that $b$ and $\theta$ are not constants. Suppose that

$$
F(s)=\sum_{n=1}^{\infty} a_{n} n^{-s} \quad(s=\sigma+i t, \sigma>1)
$$

can be continued analytically to the right of the Hooley-Huxley contour (we explain the Hooley-Huxley contour in the next paragraph) (except possibly for an isolated singularity at $s=1$ ) and in $|s-1| \geq$ a suitable positive constant, $F(s)=O_{\eta}\left(T^{\eta}\right)$ for every $\eta>0$. (The conditions $\sigma^{\prime} \leq \theta$ and $\sigma^{\prime}>\theta$ following (22) of [2] should read $\sigma \leq \theta$ and $\sigma>\theta$ respectively.)

We take the rectangle $1 / 2 \leq \sigma \leq 1,|t| \leq T+20000(\log T)^{2}$ and divide it into equal rectangles of height $100(\log T)^{2}$ (the smaller rectangles at the ends we ignore) seeing that the real line cuts into two equal portions one of these rectangles $R_{0}$. Let $R^{n}\left(n=-n_{1}, \ldots, n_{1}\right)$ be these rectangles. In a typical rectangle $R^{n}$ (with $|n|<n_{1}$ ) we fix a new right side and obtain a new rectangle $R_{n, 0}$ as follows. Take $R^{n-1}, R^{n}, R^{n+1}$ whenever all are defined and in the union of these rectangles, pick out a zero $\varrho_{n}$ with greatest real part $\beta_{n}$ of the series $F(s)$. On $R^{n}$, we shall fix the new right side $\sigma=\beta_{n}$ instead of $\sigma=1$. Consider only the right edges of these rectangles and join the ends of these edges by horizontal lines. These form the contour with the change that the contour shall not cross the real line but shall traverse from 
$\beta_{0}$ below the real axis; then it makes a circular detour round the point 1 and comes back to $\beta_{0}$ above the real axis.

If $\beta_{n}<\theta$ then, in place of $\beta_{n}$, we shall take $\beta_{n}^{\prime}=\beta_{n}+3 a\left(1-\beta_{n}\right)$. If $\beta_{n}>\theta$, then in place of $\beta_{n}$, we shall take $\beta_{n}^{\prime}=\beta_{n}+b\left(1-\beta_{n}\right)$. This contour will work for the proof of the main theorem. If $C_{0}^{\prime}$ denotes the contour from $\beta_{0}^{\prime}$ below the real line making a circular detour round the point 1 and coming back to $\beta_{0}^{\prime}$ above the real axis, then it is clear from Cauchy's theorem and the fact $\left(1-\beta_{0}\right)^{-1}=O\left((\log \log T)^{10}\right)$ that

$$
\int_{C_{0}^{\prime}} F(s) \frac{(x+h)^{s}-x^{s}}{s} d s=\int_{0}^{h} d u\left(\int_{C_{0}^{\prime}} F(s)(x+u)^{s-1} d s\right)
$$

is the same as $C_{0}^{\prime}$ replaced by $C_{0}$ with an error $O\left(h \exp \left(-(\log x)^{1 / 2}\right)\right)$ provided $T$ is chosen to be a positive constant power of $x$.

We will denote the modified contour by $M$. It consists of $C_{0}^{\prime}$ and the portion $M_{1}$ which lies strictly above the real axis and the portion $M_{2}$ which lies strictly below the real axis. We divide the contour $M_{1}$ into three parts $M_{1,1}, M_{1,2}, M_{1,3}$ according as $\sigma \leq \theta, \theta<\sigma \leq \theta+b(1-\theta), \sigma>\theta+b(1-\theta)$. Similarly for $M_{2}$ we have three parts $M_{2,1}, M_{2,2}, M_{2,3}$. Let

$$
G(u)=\int_{M-C_{0}^{\prime}} F(s) u^{s-1} d s .
$$

We assume that

$$
|F(s)| \leq \exp \left((\log T)^{\psi}\right) \quad(\psi, 0<\psi<1 \text { is any absolute constant })
$$

on $M_{1,1}, M_{1,2}, M_{2,1}$ and $M_{2,2}$. Also we assume

$$
|F(s)| \leq \exp \left((\log T)^{\psi^{\prime}}\right) \quad\left(\psi^{\prime}, 0<\psi^{\prime}<1 / 5 \text {, is any absolute constant }\right)
$$

on $M_{1,3}$ and $M_{2,3}$. With these we have the following contributions to $G(u)$ $(x \leq u \leq x+h)$.

(i) From $\sigma \leq \theta$

$$
\text { (1) } \begin{aligned}
O\left(\exp \left((\log T)^{\psi}\right)\right. & \left.\left(\frac{T^{B(1-\sigma)}}{x^{1-\sigma^{\prime}}}\right)(\log T)^{A}\right) \\
& \leq \exp \left(2(\log T)^{\psi}\right)\left(\frac{T^{B(1-3 a)^{-1}}}{x}\right)^{(1-3 a)(1-\theta)}
\end{aligned}
$$

(provided $T^{B(1-3 a)^{-1}} \leq x^{1-\delta}$ for some small positive constant $\delta$ )

$$
\leq \exp \left(2(\log T)^{\psi}\right) \exp (-\delta(\log x)(1-3 a)(1-\theta)) \text {. }
$$


(ii) From $\theta<\sigma<\theta+b(1-\theta)$

(2) $O\left(\exp \left((\log T)^{\psi}\right)\left(\frac{T^{167(1-\sigma)^{3 / 2}}}{x^{1-\sigma^{\prime}}}\right)(\log T)^{A}\right)$

$$
\begin{aligned}
& \leq \exp \left((2 \log T)^{\psi}\right)\left(x^{-1} T^{167(1-b)^{-3 / 2}\left(1-\sigma^{\prime}\right)^{1 / 2}}\right)^{1-\sigma^{\prime}} \\
& \leq \exp \left(2(\log T)^{\psi}\right)\left(x^{-1} T^{167(1-\theta)^{1 / 2}(1-b)^{-1}}\right)^{(1-b)(1-\theta)}
\end{aligned}
$$

(provided $T^{167(1-\theta)^{1 / 2}(1-b)^{-1}} \leq x^{1 / 2}$ )

$$
\leq \exp \left(2(\log T)^{\psi}\right) \exp \left(-\frac{1}{2}(\log x)(1-b)(1-\theta)\right) .
$$

(iii) From $\sigma>\theta$

(3) $\quad O\left(\exp \left(2(\log T)^{\psi^{\prime}}\right)\left(x^{\sigma^{\prime}-1} T^{167(1-\sigma)^{3 / 2}}\right)(\log T)^{A}\right)$

$$
\begin{aligned}
& \leq \exp \left(4(\log T)^{\psi^{\prime}}\right)\left(x^{-1} T^{167(1-b)^{-3 / 2}\left(1-\sigma^{\prime}\right)^{1 / 2}}\right)^{1-\sigma^{\prime}} \\
& \leq \exp \left(4(\log T)^{\psi^{\prime}}\right)\left(x^{-1} T^{167(1-\theta)^{1 / 2}(1-b)^{-1}}\right)^{(1-b) \alpha_{0}(\log T)^{-4 / 5}}
\end{aligned}
$$

(provided $T^{167(1-\theta)^{1 / 2}(1-b)^{-1}} \leq x^{1 / 2}$ and $\alpha_{0}$ is a suitable absolute positive constant)

$$
\leq \exp \left(4(\log T)^{\psi^{\prime}}\right) \exp \left(-\frac{1}{2}(\log x)(1-b) \alpha_{0}(\log T)^{-4 / 5}\right) .
$$

Hence if $T^{B(1-3 a)}=x^{1-\delta}$, we have the total contribution

$$
O\left(h \exp \left(-(\log x)^{1 / 6}\right)+x^{\varphi}\right)
$$

where $\varphi=1-1 / B+\varepsilon$. The term $x^{\varphi}$ comes from Lemma 2 of [2].

Thus we state our

MaIn Theorem. Let $\varphi=1-1 / B+\varepsilon$ (where $\varepsilon>0$ is an arbitrary constant), $1 \leq h \leq x$, and $N(x)=\sum_{n \leq x} a_{n}$. Then

$$
N(x+h)-N(x)=I(x, h)+O\left(h \exp \left(-(\log x)^{1 / 6}\right)+x^{\varphi}\right) .
$$

Let $\varphi^{\prime}=1-2 / B+\varepsilon$ (where $\varepsilon$ is as before $), 1 \leq h=h(X) \leq X(\log X)^{-A}$ where $A \geq 10$ is any constant. Then

$$
\frac{1}{X} \int_{X}^{2 X}|N(x+h)-N(x)-I(x, h)|^{2} d x=O\left(h^{2} \exp \left(-(\log X)^{1 / 6}\right)+X^{2 \varphi^{\prime}}\right) .
$$

Here, as in Ramachandra's paper,

$$
I(x, h)=\frac{1}{2 \pi i} \int_{0}^{h}\left(\int_{C_{0}} F(s)(v+x)^{s-1} d s\right) d v,
$$

where $C_{0}$ is a circular contour with centre 1 and small constant as radius with its point of intersection with the real axis to the left of 1 being removed and the integration is done in the anti-clockwise direction. 
Remark 1. The terms $h \exp \left(-(\log x)^{1 / 6}\right)$ and $h^{2} \exp \left(-(\log X)^{1 / 6}\right)$ in the $O$-terms can be replaced by $h \exp \left(-(\log x)^{1 / 3}(\log \log x)^{-10}\right)$ and $h^{2} \exp \left(-(\log X)^{1 / 3}(\log \log X)^{-10}\right)$ respectively. Also we can allow $\psi^{\prime}$ to be any positive constant $<1 / 3$.

Remark 2. The first part of the theorem is proved with some details. The second part can be proved similarly.

3. Special conditions and working examples. In general we can take $F(s)$ to be $F_{1}(s)$ as before. But we point out that we can tackle the following more general situation.

(i) $P_{1}^{\prime}=\prod L^{\nu^{-s} k}$ where the product is finite and runs over triplets $L, \nu, k$ ( $\nu \geq 1$ integer and $k$ complex $)$.

(ii) $P_{2}^{\prime}=\prod\left(L^{(j)}\right)^{k}$ where the product is finite and runs over triplets $L, j, k\left(k \geq 0\right.$ integer, $j=+1,+2, \ldots$ where $L^{(j)}(j>0)$ is the $j$ th derivative and $L^{(j)}(j<0)$ is $\left.\sum_{n=2}^{\infty}(\log n)^{-j} n^{-s}\right)$.

(iii) $P_{3}^{\prime}=\prod(\log L)^{k}$ where the product is finite and runs over pairs $L, k$ $(k \geq 0$ integer $)$ and $F(s)=P_{1}^{\prime}(s) P_{2}^{\prime}(s) P_{3}^{\prime}(s) F_{0}(s)$.

The necessary estimates for $F(s)$ are provided by Lemma 5 of [2]. (It has to be pointed out that in the proof of Lemma 5 of [2], $\sigma$ does not denote the real part of $s$. Also on page 322 of [2] in the proof, the line 7 from the top should read $(1-\sigma)^{-1}=O(\log \log T)$ and in the line 5 from the bottom, the $O$-condition should read $(1-\sigma)^{-1}=O(\log T)$.) The only change in the lemma is (since now $b$ is not a constant)

$$
\log L(s)=O\left((\log T)^{(1-d)(1-2 a)^{-1}}(\log \log T)^{4}\right)
$$

and the remark below Lemma 5 of [2] applies without any change.

Now we need the bound for the coefficients $a_{n}=a_{n}(x)$. For this purpose we prove some lemmas. Except Lemma 1, all are routine.

LEMMA 1. Let $h>1$ be any integer and

$$
(\zeta(s))^{h^{-s}}=\sum_{n=1}^{\infty} b_{n} n^{-s} .
$$

Then $b_{1}=1$ and for $n>1$ we have $0 \leq b_{n} \leq \log n$.

Proof. We have

$(\zeta(s))^{h^{-s}}=\exp \left(h^{-s} \sum_{m \geq 1} \sum_{p}\left(m p^{m s}\right)^{-1}\right)=1+\sum_{\nu=1}^{\infty}\left(h^{-s} \sum \sum \ldots\right)^{\nu}(\nu !)^{-1}$

(note that this is also valid for $h=1$ ), and writing

$$
\left(\sum \sum \ldots\right)^{\nu}=\sum_{n=1}^{\infty} d_{n}(\nu) n^{-s}
$$


we have

$$
\sum_{\nu=1}^{\infty} d_{n}(\nu)(\nu !)^{-1}=1
$$

for all $n \geq 2$ and so $d_{n}(\nu) \leq(\nu !)$. Thus for $n \geq 2$

$$
b_{n}=\sum_{m h^{\nu}=n, m \geq 2^{\nu}} d_{m}(\nu)(\nu !)^{-1}=\sum_{\nu=1, n h^{-\nu}=m, m \geq 2^{\nu}}^{\infty} d_{m}(\nu)(\nu !)^{-1} .
$$

Note that if $n h^{-\nu}<2^{\nu}$, then $m$ does not exist; i.e. if $\nu>\log n / \log (2 h)$, then $m$ does not exist. Since given a $\nu$ there is at most one $m$, we have

$$
b_{n}=\sum_{1 \leq \nu \leq \log n / \log (2 h)} d_{m}(\nu)(\nu !)^{-1} \leq \log n .
$$

LEMma 2. The product of two series of the form

$$
\sum_{n=1}^{\infty} a_{n} n^{-s}, \quad a_{n}=O_{\eta}\left((n x)^{\eta}\right)
$$

is of the same form.

Proof. Since $\eta$ is arbitrary, the lemma follows from

$$
\sum_{d d^{\prime}=n} a_{d} b_{d^{\prime}} \ll_{\eta} \sum_{d d^{\prime}=n}\left(d d^{\prime} x^{2}\right)^{\eta} \leq x^{2 \eta} n^{\eta} d(n) \ll(n x)^{10 \eta} .
$$

LEMMA 3. Let $k$ be any complex number and $u=[|k|]+1$. Then the coefficients of $(\zeta(s))^{k h^{-s}}$ (in absolute value) do not exceed those of $(\zeta(s))^{u h^{-s}}$.

R e m a r k. Using Lemmas 1, 2 and 3 we can verify the required property for $F(s)$ uniformly in certain obvious ranges for $j$ and $k$. (See also Lemma 4 to follow.)

From now on we concentrate on the special case where $a_{n}$ is defined by $(\zeta(s))^{k}=\sum_{n=1}^{\infty} a_{n} n^{-s}\left(a_{n}\right.$ are the well-known coefficients $\left.d_{k}(n)\right)$ and $k \geq 1$ is an integer. Here we deal with uniformity in $k$ subject to $1 \leq k \leq$ $\exp (o(\log \log x))$.

LEMMA 4. Let $d_{k}(n)$ be defined by

$$
(\zeta(s))^{k}=\sum_{n=1}^{\infty} d_{k}(n) n^{-s}
$$

where $\operatorname{Re} s>1$ and $k$ is any positive integer. Then uniformly for $1 \leq k \leq K$ 
where $K=\exp (o(\log \log x))$ we have

$$
d_{k}(n)=O_{\eta}\left((n x)^{\eta}\right)
$$

for $n>1$ and every constant $\eta>0$.

Proof. We have $\left(d_{k}(n)\right)^{l} \leq d_{J}(n)$ where $J=k^{l}$ and $l$ is any positive integer and so

$$
\left(d_{k}(n)\right)^{l} \leq n^{2} \sum_{m=1}^{\infty} d_{J}(m) m^{-2}=n^{2}(\zeta(2))^{J} \leq n^{2} e^{J}
$$

Thus for all $n>1$, we have

$$
\log d_{k}(n) \leq \frac{2 \log n}{l}+\frac{k^{l}}{l} \leq \frac{2 \log n}{l}+\frac{(\log x)^{\eta^{2} l}}{l}
$$

for all large $x$. Putting $l=\left[\eta^{-1}\right]$ we obtain

$$
\log d_{k}(n) \leq 3 \eta \log n+3(\log x)^{\eta} .
$$

Replacing $\eta$ by $\eta / 3$, we obtain $d_{k}(n) \ll(n x)^{\eta}$ for all $x \geq x_{0}(\eta)$. This proves the lemma.

We now resume the case $F(s)=(\zeta(s))^{k}=\sum_{n=1}^{\infty} a_{n} n^{-s}$ where $a_{n}=$ $d_{k}(n)$. We only have to obtain an asymptotic formula for

$$
I(x, h)=\int_{0}^{h}\left(\frac{1}{2 \pi i} \int_{C_{0}}(\zeta(s))^{k}(v+x)^{s-1} d s\right) d v .
$$

Now since the expansion of $\zeta(s)(s-1$ ) (in powers of $s-1$ ) converges for $|s-1| \leq 10$ we have

$$
\zeta(s)(s-1)=1+\nu_{1}(s-1)+\nu_{2}(s-1)^{2}+\ldots
$$

where $\nu_{j}$ is a sequence of bounded real numbers. Hence

$$
(\zeta(s)(s-1))^{k}=1+\nu_{1}^{(k)}(s-1)+\nu_{2}^{(k)}(s-1)^{2}+\ldots
$$

where $\left|\nu_{j}^{(k)}\right|$ is majorized by the coefficient of $(s-1)^{j}$ in the expansion of $(1-C(s-1))^{-k}$ for a suitable constant $C>1$. Thus

$$
\left|\nu_{1}^{(k)}\right| \leq C k, \quad\left|\nu_{2}^{(k)}\right| \leq C^{2} \frac{k(k+1)}{2}, \ldots
$$

Now, writing $y$ for $v+x$, we have by Cauchy's theorem

(5) $\frac{1}{2 \pi i} \int_{C_{0}}(\zeta(s))^{k} y^{s-1} d s=\frac{(\log y)^{k-1}}{(k-1) !}+\nu_{1}^{(k)} \frac{(\log y)^{k-2}}{(k-2) !}+\ldots+\nu_{k}^{(k)}$. 
The left-hand side of (5) is

$$
\begin{gathered}
\frac{(\log y)^{k-1}}{(k-1) !}\left(1+O\left(\frac{(k-1) C k}{\log y}+\frac{(k-1)(k-2) C^{2} k(k+1)}{2 !(\log y)^{2}}+\ldots\right.\right. \\
\left.\left.+\frac{C^{k} k(k+1) \ldots(2 k)}{k !(\log y)^{k}}\right)\right) \\
=\frac{(\log y)^{k-1}}{(k-1) !}\left(1+O\left((\log y)^{-1 / 2}\right)\right)
\end{gathered}
$$

provided $k=O\left((\log y)^{1 / 4}\right)$. In fact, since we need the restriction $k \leq$ $\exp (o(\log \log x))$ this is amply satisfied. This completes the proof of the following Theorem 4 which is a corollary to the main theorem.

THEOREM 4. The main theorem is true with $F(s)=(\zeta(s))^{k}$ uniformly for positive integers $k \leq \exp (o(\log \log x))$ for the first result and $k \leq$ $\exp (o(\log \log x))$ for the second result. Moreover,

$$
\begin{aligned}
I(x, h) & =\int_{0}^{h}\left(\frac{1}{2 \pi i} \int_{C_{0}}(\zeta(s))^{k}(v+x)^{s-1} d s\right) d v \\
& =\int_{0}^{h} \frac{(\log (v+x))^{k-1}}{(k-1) !}\left(1+O\left((\log (v+x))^{-1 / 2}\right)\right) d v \\
& =\frac{h(\log x)^{k-1}}{k-1 !}\left(1+O\left((\log x)^{-1 / 2}\right)\right) .
\end{aligned}
$$

Remark 1. First of all, we note that the implied constant in the theorem has to depend on $\varepsilon$ and $\eta$. The only thing to be checked to conclude the last step is

$$
(\log (v+x))^{k-1}=(\log x)^{k-1}+O\left((\log x)^{k-1-1 / 2}\right) .
$$

This is true because the $O$-term is really $(\operatorname{since} \log (v+x)-\log x=O(1))$

$$
\begin{aligned}
\sum_{r=1}^{k-1}(k & -1)_{C_{r}}(\log x)^{k-1-r}(O(1))^{r} \\
& \leq(\log x)^{k-1}(\log x)^{-1 / 2} \sum_{r=0}^{k-1}(k-1)_{C_{r}}(\log x)^{-r / 2}(O(1))^{r} \\
& =(\log x)^{k-1-1 / 2}\left(1+O\left((\log x)^{-1 / 2}\right)\right)^{k}=O\left((\log x)^{k-1-1 / 2}\right) .
\end{aligned}
$$

This completes the proof of Theorem 4 .

Remark 2. It is not hard to prove that if $1 \leq k \leq \exp (o(\log \log x))$, 
then

$$
I(x, h)=\frac{h(\log x)^{k-1}}{(k-1) !}\left(1+O_{\eta}\left((\log x)^{-1+\eta}\right)\right) .
$$

Acknowledgements. The authors are indebted to Professors R. Balasubramanian and K. Ramachandra for initiation to the subject, helpful discussions and encouragement. The second author is thankful to the National Board for Higher Mathematics for its financial support.

\section{References}

[1] I. Kátai, A remark on a paper of Ramachandra, in: Number Theory, Proc. Ootacamund, K. Alladi (ed.), Lecture Notes in Math. 1122, Springer, 1984, 147-152.

[2] K. Ramachandra, Some problems of analytic number theory, Acta Arith. 31 (1976), 313-324.

SCHOOL OF MATHEMATICS

TATA INSTITUTE OF FUNDAMENTAL RESEARCH

HOMI BHABHA ROAD

BOMBAY 400005

INDIA

Received on 7.1.1992

and in revised form on 26.5.1992 